Open Access

\title{
In ovo administration of human recombinant leptin shows dose dependent angiogenic effect on chicken chorioallantoic membrane
}

Reji Manjunathan and Malathi Ragunathan ${ }^{*}$

\begin{abstract}
Background: Leptin, the cytokine produced by white adipose tissue is known to regulate food energy homeostasis through its hypothalamic receptor. In vitro studies have demonstrated that leptin plays a major role in angiogenesis through binding to the receptor Ob-R present on ECs by stimulating and initiating new capillary like structures from ECs. Various in vivo studies indicate that leptin has diverse effect on angiogenesis. A few reports have showed that leptin exerts pro angiogenic effects while some suggested that it has antiangiogenic potential. It is theoretically highly important to understand the effect of leptin on angiogenesis to use as a therapeutic molecule in various angiogenesis related pathological conditions. Chicken chorio allantoic membrane (CAM) on 9th day of incubation was incubated with 1, 3 and $5 \mu \mathrm{g}$ concentration of HRL for $72 \mathrm{~h}$ using gelatin sponge. Images where taken after every $24 \mathrm{~h}$ of incubation and analysed with Angioguant software. The treated area was observed under microscope and histological evaluation was performed for the same. Tissue thickness was calculated morphometrically from haematoxylin and eosin stained cross sections. Reverse transcriptase PCR and immunohistochemistry were also performed to study the gene and protein level expression of angiogenic molecules.

Results: HRL has the ability to induce new vessel formation at the treated area and growth of the newly formed vessels and cellular morphological changes occur in a dose dependent manner. Increase in the tissue thickness at the treated area is suggestive of initiation of new capillary like structures. Elevated mRNA and protein level expression of VEGF165 and MMP2 along with the activation of ECs as demonstrated by the presence of CD34 expression supports the neovascularization potential of HRL.

Conclusion: Angiogenic potential of HRL depends on the concentration and time of incubation and is involved in the activation of ECs along with the major interaction of VEGF 165 and MMP2. It is also observed that $3 \mu \mathrm{g}$ of HRL exhibits maximum angiogenic potential at $72 \mathrm{~h}$ of incubation. Thus our data suggest that dose dependent angiogenic potential HRL could provide a novel role in angiogenic dependent therapeutics such as ischemia and wound healing conditions.
\end{abstract}

Keywords: Angiogenesis, HRL, Human Recombinant Leptin, CAM, chorioallantoic membrane, ECs, endothelial cells, VEGF, vascular endothelial growth factor, MMP, matrix metalloproteinase, Blood vessels, CD34

\footnotetext{
*Correspondence: rmalathigenetics@gmail.com

Department of Genetics, Dr. ALM PG IBMS, Taramani Campus, University of

Madras, Chennai 600 113, Tamilnadu, India
} 


\section{Background}

The $\mathrm{Ob}$-gene product leptin is $16 \mathrm{kDa}$ peptide hormone, produced mainly by white adipose tissue is proposed to play a key role in the regulation of body weight and thermogenesis through the receptor found in the hypothalamus [1]. Accumulating evidences from in vitro and in vivo assays suggest that leptin promote endothelial cell (EC) proliferation and survival in favour of angiogenesis [2,3]. In vivo and in vitro results from Hyung et al. study indicates that angiogenic potential of leptin is mediated by matrix metalloproteinase (MMPs) [4]. However, the actual mechanism behind the involvement of matrix metalloproteinase in leptin mediated angiogenesis is not clear.

The effect of leptin on angiogenesis is still not understood as some reports have suggested its potential to induce angiogenesis while some have indicated of its anti angiogenic effect [5-9]. Recently it has been reported that in ovo administration of leptin inhibited angiogenesis on chicken chorio allantoic membrane (CAM) [10]. Leptin has been administered as a therapeutic molecule in various angiogenesis related pathological conditions especially in wound healing [11]. However a quantitative in vivo evaluation on the effect of leptin on angiogenesis is important because of its therapeutic application potential in the pathology of angiogenesis dependent conditions. Therefore in the present study we examined the angiogenic potential of human recombinant leptin (HRL) using well vascularized CAM of developing chicken embryo (Gallus gallus domesticus). The advantage of CAM assay is that it is a highly vascularized structure with potential growth and this in ovo vivo system is highly useful to understand the physiological angiogenesis and hence widely used for the screening of various compounds for their angiogenic activity [12].

In the present study we analysed and compared the angiogenic ability of HRL of varying concentrations such as 1,3 and $5 \mu \mathrm{g}$ for an incubation period of $72 \mathrm{~h}$ using late CAM. Gelatin sponges soaked with leptin were placed on the membrane at 9th day of post incubation so as to allow slow delivery of the chemical at the treated area with less or no inflammation $[13,14]$. The ability of HRL to induce new vessel growth at the treated area is directly visualized from the CAM images taken at different time period of incubation and growth of these vessels measured with Image $J$ and Angioquant MATLAB softwares [14-16]. Angiogenic response of HRL at the treated area is further analysed in detail from the histological sections. We also examined the expression of major angiogenic molecules at the molecular and protein level to understand the involvement of these factors on HRL induced angiogenesis. Our findings suggest that the potential of HRL to induce angiogenesis depends on various physiological factors especially dose and time of incubation. The result have demonstrated that HRL favours neovascularization through sprouting of vessels which is accelerated by the expression of VEGF165 and MMP2 in a dose dependent way in chicken CAM vasculature.

\section{Methods \\ Materials}

Fertilized white leghorn chicken eggs were purchased from Tamil Nadu Poultry Research Station, Madras Veterinary University, Nandanam, Tamil Nadu, India. Gelatin sponges purchased from Jhonson \& Jhonson Pvt Lmtd, India, Paraffin film and wax were purchased from Sigma, Aldrich, USA. Haematoxylin and Eosin stain purchased from Medox, India and Human recombinant leptin from MP Biomedicals, Inc. France. TRIzol reagent, agarose and EtBr were from Sigma, Aldrich, USA. ImProm-1 ${ }^{\mathrm{rm}}$ Reverse Transcriptase kit and GoTaq Green Master Mix PCR amplification kit were from Promega, USA, Oligo (dt) of length 18-meres from eurofins, mwg operon, Germany, Random hexamers from MP Biomedicals, USA. All primers were purchased from Bioserve, India. DNA ladders purchased from Invitrogen, USA. DAB system purchased from Bangalore Genei, India. CD34 antibody (Endothelial Cell Marker, Cluster designation 34) from US Biological, USA and VEGF A from CALBIOCHEM, EMD. Bradford reagent and FITC (Goat ant-rabbit IgG were from Bangalore Genei, India. SDSPAGE Standards marker was from BIO-RAD, CANADA and gelatin from Medox, India. Rabbit polyclonal MMP2 is a kind gift from Dr. Li Haiqing, MD, Ph.D, -Technology transfer specialist, National Cancer Institute, Rockville, USA. Unless otherwise specified all other common reagents and chemicals were purchased from Sigma, Aldrich, USA.

\section{The in ovo CAM model}

Fertilized white leghorn chicken eggs weighing $50 \pm 2$ g, were incubated at $37{ }^{\circ} \mathrm{C}$ in a 'humidified atmosphere ( $>60 \%$ relative humidity) as per the protocol described in Hen's Egg Test - Chorioallantoic Membrane (HETCAM) method adapted from ZEBET (The German Centre for the Documentation and validation of Alternative Methods, Republic of Germany). At day 3 of post incubation, 2 to $3 \mathrm{ml}$ of albumin was withdrawn, using a 21 gauge needle, through the large blunt edge of the egg in order to minimize the adhesion of the shell membrane with CAM. A square window of $2 \mathrm{~cm}^{2}$ was opened in the egg shell and sealed with paraffin film to prevent dehydration and the eggs were incubated further. At day 9 of post incubation, gelatin sponges of size of $1 \mathrm{~mm}^{3}$ were placed on top of the growing CAM under sterile condition $[14,17]$ and were soaked with $15 \mu \mathrm{l}$ volume of 1,3 and $5 \mu \mathrm{g}$ concentration of HRL. Control eggs were incubated with 1X PBS (pH-7.3). The window was closed with a 
transparent adhesive tape and eggs were incubated for $72 \mathrm{~h}$ until it reached post incubation day 12. CAM were photographed at 0, 24, 48 and $72 \mathrm{~h}$ using Canon digital camera and images were analysed with Image J and Angioquant Toolbox, MATLAB 6.5 software to measure total length and size of the blood vessels (micrometre) from the area of treatment.

\section{Light microscope analysis}

After $72 \mathrm{~h}$ of incubation the area of the CAM treated with HRL was detached carefully. The excised membrane was kept on glass slides and images were taken using light microscope both at 4 and $10 \times$ magnification to view the growth of capillaries [18].

\section{Histology}

After $72 \mathrm{~h}$ of incubation area of the CAM treated with HRL was flooded with Bouin's fixative solution. Around $1 \mathrm{~cm}^{2}$ of the membrane around the treated area was removed carefully using forceps and surgical scissors and dehydrated through graded series of alcohol (50\%, $70 \%$, $90 \%$ and absolute) and embedded in paraffin wax. Vertical cross tissue sections ( $7 \mu \mathrm{m}$ in thickness) were taken using Rotary Microtome (Weswicox, Japan). Sections were treated with alcohol in ascending order (absolute, $90 \%$, $70 \%$, and $50 \%$ ) and cleared with xylene before staining with haematoxylin and eosin. After mounting with DPX, the histological sections were observed under light microscope at 40× magnification for qualitative assessment and images were recorded using Nikon Camera attached with light microscope at 10× magnification [14].

\section{Morphometric analysis of CAM tissue thickness (DCAM)}

Thickness of the CAM for all the groups treated with HRL were measured from haematoxylin and eosin stained vertical cross sections using a calibrated objective at $40 \times$ magnification with $10 \times 10$ calibrated grid at $10 \times$ ocular. Distances between the chorionic and allantoic epithelial layers were measured in micrometre at 6 different locations from the same sample and is repeated for six serial cross sections of the same. Average tissue thickness was calculated from each tissue sample of the same and obtained a mean DCAM thickness $[14,19]$.

\section{Semi-Quantitative Reverse Transcriptase-Polymerase Chain Reaction (RT-PCR)}

Total RNA was isolated from CAM treated with HRL using TRIzol reagent $(100 \mathrm{mg} / 1 \mathrm{ml})$. The quantity and the purity of the isolated RNA were checked using UVvisible spectrophotometer. cDNA of $20 \mu \mathrm{l}$ volume was synthesized using ImProm- $11^{\text {Tw }}$ Reverse Transcriptase kit with Oligo (dt) of length 18-meres and random hexamers. PCR amplification was performed using GoTaq Green Master Mix kit and changes in the level of mRNA expression of VEGF165 [20], VEGF121 [21], MMP2 [20], MMP9 [22] and GAPDH [23] were evaluated using PCR with 100 pico moles of specific primers. The relative expression level of each mRNA transcript was normalized with that of control. PCR products were subjected to electrophoresis.

\section{Gelatin zymography}

CAM tissues at the treated area from each group were homogenized $(100 \mathrm{mg} / \mathrm{ml})$ using Tris buffer $(0.5 \mathrm{M}$ Tris-HCl (pH-6.8), $10 \%$ SDS, glycerol and $0.01 \%$ bromophenol blue) and centrifuged at $12,000 \mathrm{rpm} / 4{ }^{\circ} \mathrm{C} /$ $10 \mathrm{~min}$. The concentration of protein from the supernatant was determined using Bradford reagent and the gelatinase activity was examined on a $10 \%$ SDS-PAGE electrophoresis containing $1.0 \mathrm{mg} / \mathrm{ml}$ of gelatin. Protein samples of $25 \mu \mathrm{g} / 40 \mu \mathrm{l}$ were loaded per well along with $20 \mu \mathrm{l}$ pertained SDS-PAGE Standards marker. After electrophoresis, the gels were washed with $2.5 \%$ of Triton-X-100 and incubated in digestion buffer $(50 \mathrm{mM}$ Tris HCl-pH-7.5,100 mM CaCl2, $1 \mu \mathrm{M} \mathrm{ZnCl2,} 1 \%$ Triton X-100, $0.02 \% \mathrm{NaN} 3-100 \mathrm{ml}$ ) for 16 to $18 \mathrm{~h}$ at $37^{\circ} \mathrm{C}$ with gentle agitation. The gel was stained with staining solution $(0.05 \%(w / v$, Coomassie blue in $50 \%$ methanol and $10 \%$ acetic acid) for $1 \mathrm{~h}$ and de stained with methanol/acetic acid mixture. Gelatinase activity of MMP2 was detected as clear white bands against background. The gels were scanned and images were recorded using BIO-RAD Calibrated Densitometer Software (GS 800, USA). The density of the bands was calculated with PD Quest Advances Software and normalized with control value [24].

\section{Immunohistochemistry}

Three different concentration namely 1,3 and $5 \mu \mathrm{g}$ of HRL have been used of which $3 \mu \mathrm{g}$ of HRL yielded maximum angiogenic response when compared to other two concentrations. The deparafinized and dehydrated CAM of $5 \mu \mathrm{M}$ thickness after treating with $3 \mu \mathrm{g}$ of HRL was allowed to undergo antigen retrieval process using Sodium Citrate (10 mM-pH 6.0) in a microwave oven for 20 min followed by washing in DDH2O for $3 \mathrm{X} 5 \mathrm{~min}$ in 1X PBS (pH 7.3). Normal Goat Serum Blocking Solution (2 \% goat serum, $1 \%$ BSA, $0.1 \%$ cold fish skin gelatin, $0.1 \%$ Triton X-100, $0.05 \%$ Tween- 20, $0.05 \%$ Sodium Azide, 0.01 M PBS (pH 7.2) of 50 to $75 \mu \mathrm{l}$ was added immediately on the sections and incubated for $1 \mathrm{~h}$ in a humidified chamber. After washing with $1 \mathrm{X}$ PBS, primary antibodies of MMP2 (1:200), VEGF (VEGF A) (1:100) and CD34 (1:200) diluted in blocking serum were applied on the sections and after overnight incubation rinsed with $1 \mathrm{X}$ PBS with $0.05 \%$ of Tween-20. Diluted FITC (Goat ant-rabbit IgG) and HRP (both Goat antirabbit and Goat anti- mouse $\operatorname{IgG}$ ) secondary antibodies 
of 1:40 dilution was applied for $1 \mathrm{~h}$ according to manufacturer's instruction. For HRP conjugated secondary antibodies DAB system was used for colour development. The slides were finally counterstained with Mayer's haematoxylin and mounted with $90 \%$ of glycerol. For HRP conjugated system the images were recorded using light microscope and for FITC conjugation BX51 Olympus Fluorescence Microscope at a wavelength of $515 \mathrm{~nm}$ with ASI FISH View 5.5 software at 40× magnification [25].

\section{Data analysis and statistical analysis}

All experiments were independently performed in triplicate. Data were analysed using one way ANOVA analysis of variance test and Tukey post hoc test as appropriate (Sigma stat 2.0). Data were expressed as means + S.E.M and $\mathrm{P}$-values of $* \mathrm{p}=<0.001$ and $\# \mathrm{p}=0.001$ were selected for showing statistically significant difference.

\section{Results}

Human recombinant leptin induces neo vascularization visual assessment of new blood vessel formation on CAM vascular bed and its growth

The main focus of the present study is to visualize directly the angiogenic ability of HRL to induce new vessel formation at the treated area. CAM incubated with all three concentrations of HRL shows growth of new capillary vessels at the treated area (Fig. 1a). After $72 \mathrm{~h}$ of incubation, $1 \mu \mathrm{g}$ of HRL (Fig. 1a. d) exhibited minimal growth of capillaries around the sponge and is visually similar to that of control CAM (Fig. 1a. b). Formations of new capillaries were more prominent with $3 \mu \mathrm{g}$ of HRL after $72 \mathrm{~h}$ and this is evident with development of reddishness around the sponge indicating increased vessel growth at the treated area (Fig. 1a. f). Spoke wheel pattern of allantoic vessel growth is observed for this particular concentration.

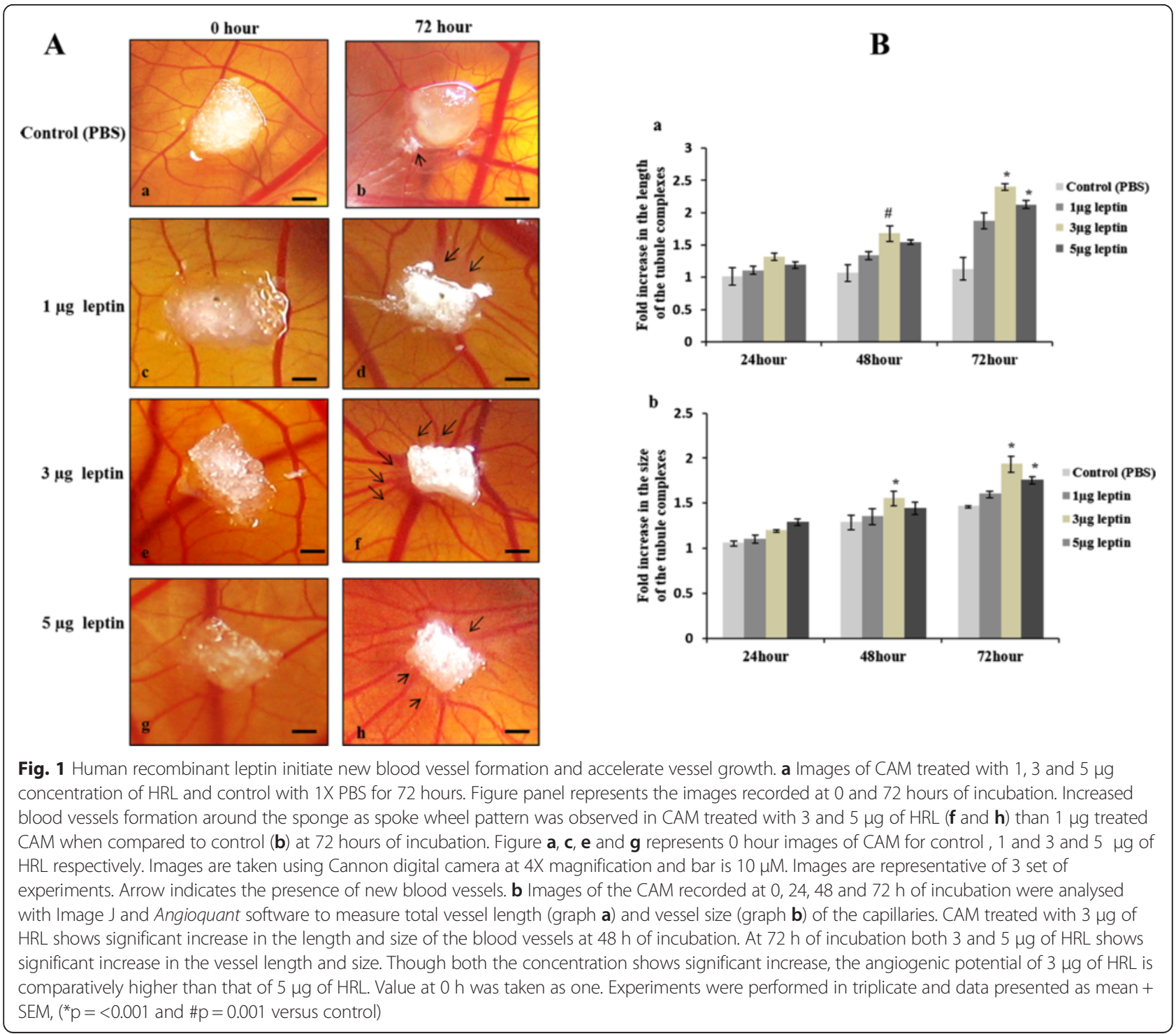


HRL of $5 \mu \mathrm{g}$ also shows numerous scattered allantoic vessels in and around sponge at $72 \mathrm{~h}$ but with lesser vessels growth when compared to that of $3 \mu \mathrm{g}$ (Fig. 1a. h).

From the above observation it can be inferred that, both 3 and $5 \mu \mathrm{g}$ concentration of HRL has the ability to induce angiogenesis through new capillary formation at $72 \mathrm{~h}$ of incubation. In order to conform this and also to arrive at the optimum working concentration for HRL we measured the growth of the vessels in terms of its length and size, from the images taken at 0, 24, 48 and $72 \mathrm{~h}$ of incubation with Image $J$ and Angioquant MATLAB software. This software is specially made for quantifying angiogenesis using in vitro assays and has been employed for CAM with some modifications using Image $J$ software $[11,13]$. Of the three different concentrations, used $3 \mu \mathrm{g}$ of HRL demonstrates a significant increase in the vessel length (Fig. 1b. b) and size (Fig. 1b. b) at $48 \mathrm{~h}$ of incubation which is 1.99 fold greater than that of control (\#p=0.001, $\left.{ }^{*} \mathrm{p}=<0.001\right)$. At $72 \mathrm{~h}$ of incubation both 3 and $5 \mu \mathrm{g}$ of HRL induces a significant increase in the vessel length and size ("p $=<0.001$ ) and of these two, $3 \mu \mathrm{g}$ of leptin shows 2.1 fold increases in vessel growth which is higher than that of $5 \mu \mathrm{g}$ that showed which has only 1.8 fold increase. HRL of $1 \mu \mathrm{g}$ also shows angiogenic potential with increase in the vessel growth up to 1.5 fold, the value is not significant when compared to control that has 1.2 fold increase in the vessel growth from 0 to $72 \mathrm{~h}$ of incubation. Thus it is obvious that HRL can induce new vessel formation at the treated area in a dose dependent manner with $3 \mu \mathrm{g}$ concentration having maximum angiogenic response.

\section{Human recombinant leptin initiate growth of vessels by means of sprouting - visualising the morphology of blood vessels on CAM vascular bed}

We analysed the morphology of blood vessels in order to confirm the sprouting angiogenic ability of HRL at the treated area. Images of the CAMs were recorded immediately after $72 \mathrm{~h}$ of incubation using microscope. It was found that HRL is able to induce growth of new blood vessels from the existing one (Fig. 2). CAM incubated with $3 \mu \mathrm{g}$ of HRL shows more number of sprouting vessels along with branches and sub branches at the treated area (Fig. 2e). An enlarged view of the same

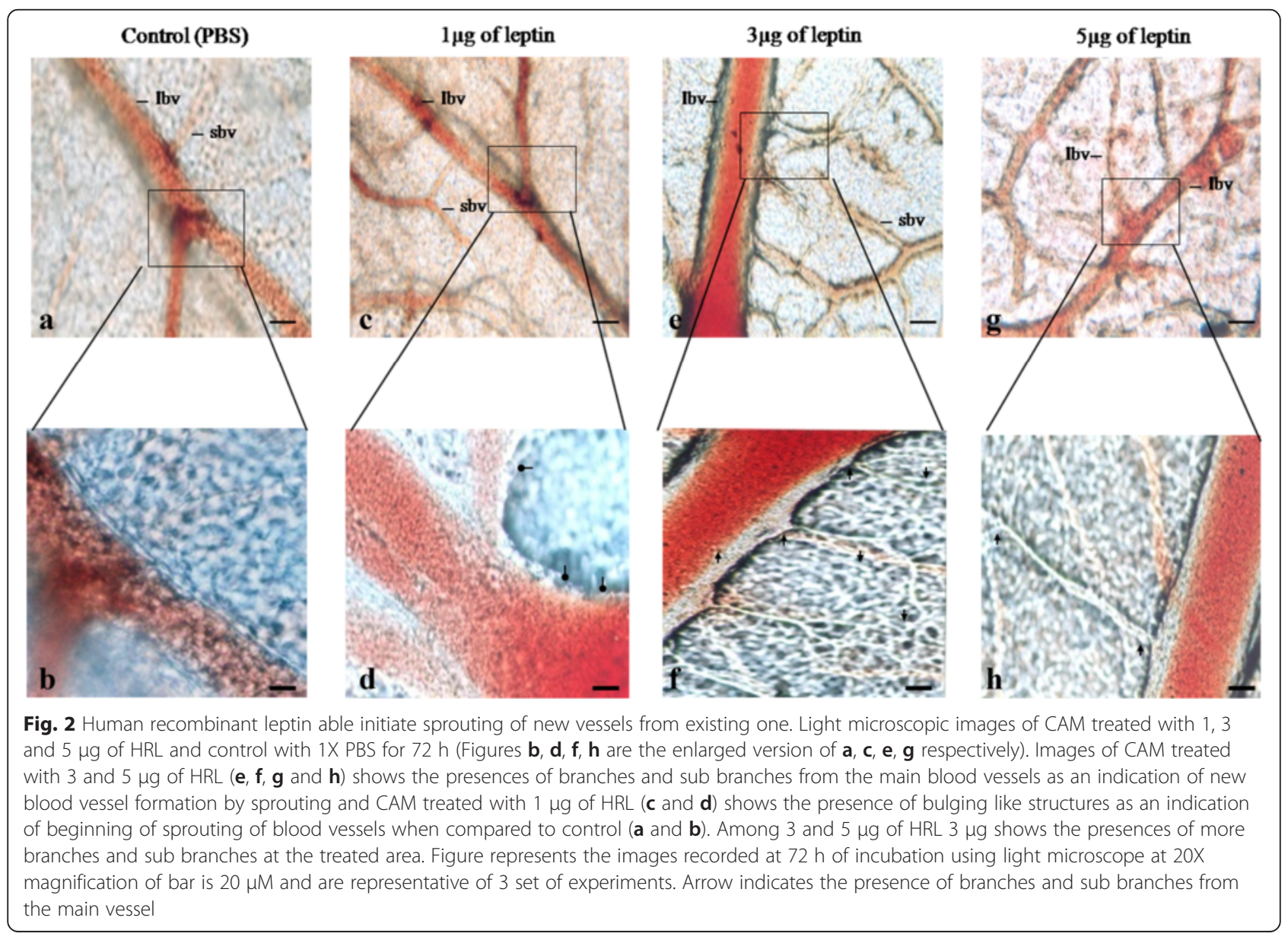


treated area shows the presence of more branches form the main vessel and sub branches from the newly formed vessels, a feature directly suggesting the fact that HRL of this particular concentration and time of incubation exerts its maximum angiogenic activity (Fig. 2f). CAM treated with $5 \mu \mathrm{g}$ of HRL also shows the presence of sprouting of vessels from the main one with appearances of branches and sub branches (Fig. 2g and h), but its potential to induce new vessel formation at the treated area is comparatively lesser than that of $3 \mu \mathrm{g}$ HRL. HRL of $1 \mu \mathrm{g}$ also shows the presence of sprouting structures from the main vessel, but is not remarkably strong when compared to other concentrations (Fig. 2c). An enlarged view of the treated area for the same shows roughness throughout the outer surface of the main blood vessel along with bulging appearance suggestive of its potential to initiate sprouting of new blood vessels at the treated area. Control CAM doesn't show any such sprout formation from the existing vessels (Fig. 2a) and the enlarged view of the same indicates stable blood vessel without any rough appearance or bud like structures from that of main one (Fig. 2b). Thus, HRL is able to induce sprouting of new vessels from the existing one with maximum sprouting efficacy at $3 \mu \mathrm{g}$ concentration.
Human recombinant leptin induces morphological changes leading to neo vascularization - histological observation of the CAM vasculature

To conform the angiogenic potential of HRL, we also analysed the changes in the cellular morphology of the CAM vasculature from cross sections of the treated area stained with haematoxylin and eosin. Changes in the morphology of the CAM vasculature has been changed observed for all three concentrations of HRL and is indicative of the positive angiogenic response due to HRL (Fig. 3). CAM incubated with $1 \mu \mathrm{g}$ of HRL (Fig. 3b) shows irregular appearance of thin stratum (which contains both chorionic (ce) and allantoic (ae) epithelial layers) with the presences of few blood vessels at the primary stratum. CAM treated with $3 \mu$ g of HRL (Fig. 3c) shows vessels enlarged in size with remarkable irregular growth pattern at thin stratum. Morphology of ECs are altered those ECs which are present at the capillary plexus/blood sinus area (which lies in between the primary and thin stratum) shows sprouting appearance typical for angiogenesis. Thickness of the primary stratum is also increased probably due to cellular accumulation at the sub epithelial capillary network (SEC). Presences of a number of small blood vessels around the main vessels is suggestive of the

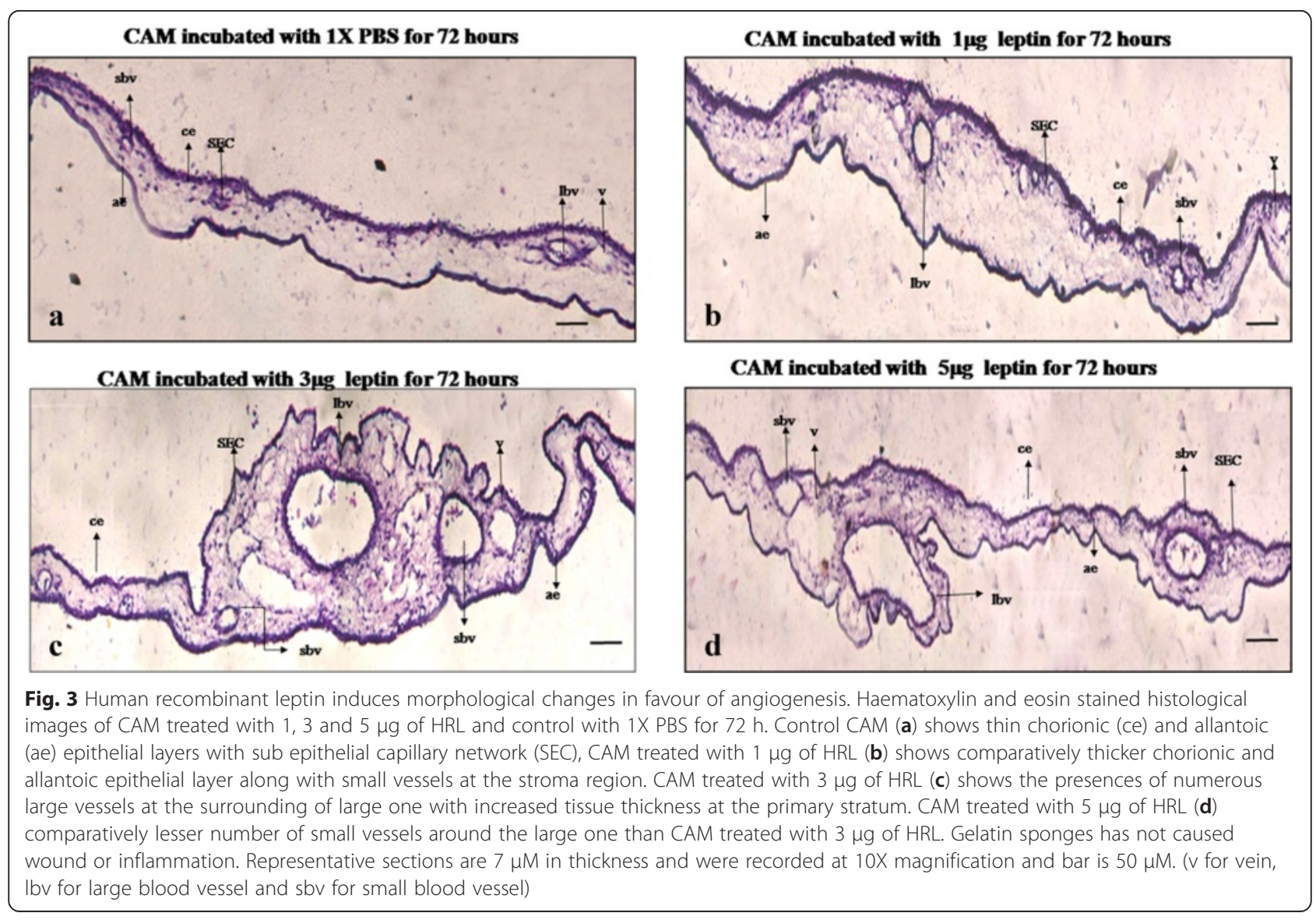


angiogenic potential of leptin either by forming new vessels or by inducing the sprouting from the main one. CAM treated with $5 \mu \mathrm{g}$ of HRL (Fig. 3d) also shows irregular appearances at thin stratum due to increase in the number of blood vessels and diminished accumulation of cells were observed beneath SEC. Very few small vessels were found around the large one as an indication of minimal sprouting which is lesser than that observed with $3 \mu$ g. Control CAM (Fig. 3a) shows a few scattered blood vessels at primary stratum with uniform growth at the thin stratum due to growth of less number of blood vessels at this area. These observations demonstrates that HRL is able to induce formation of new vessel growth by inducing positive morphological changes at the cellular level in favour of angiogenesis. Leptin of $3 \mu \mathrm{g}$ concentration is capable of eliciting promising cellular response that could lead to more angiogenic growth.

Human recombinant leptin increases the size of tissue by means of inducing new vessel growth. Quantitative measurement of the angiogenic potential of human recombinant leptin by morphometric measurement of CAM tissue thickness (DCAM)

The ability of 1,3 and $5 \mu \mathrm{g}$ of HRL to induce new vessels growth was further confirmed by measuring the size of the tissue from the from haematoxylin and eosin stained paraffin embedded vertical cross sections and is plotted as average tissue thickness (Fig. 4). Formation of new blood vessels or enlargement or growth of the existing blood vessels will cause an increase in the thickness of the CAM at the stroma and will push both the

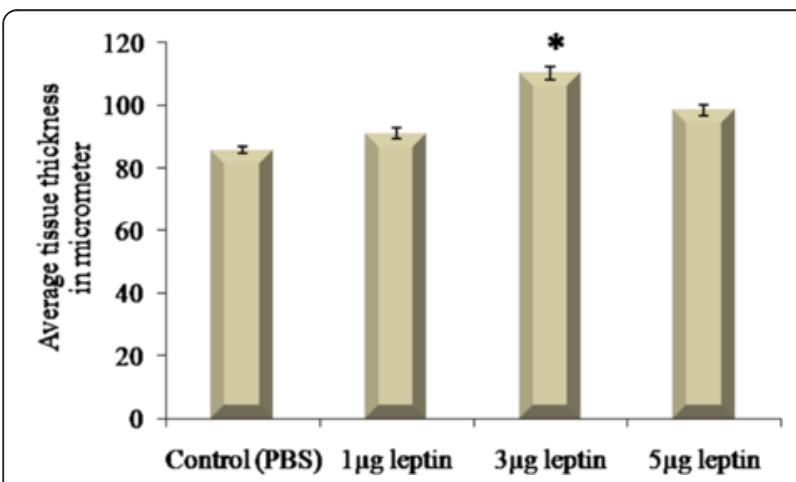

Fig. 4 Human recombinant leptin increases the size of tissue by means of inducing new vessel growth. Total thickness of the tissue at the treated area with 1, 3 and $5 \mu \mathrm{g}$ of HRL was measured from haematoxylin and eosin stained vertical cross section after $72 \mathrm{~h}$. Control CAM was treated with 1X PBS. The distance between chorionic and allantoic epithelial layers was calculated morphometrically in $\mu \mathrm{M}$. CAM treated with $3 \mu \mathrm{g}$ of HRL shows significantly increased tissue thickness than other two concentrations. Material shrinkage is estimated to be $\sim 25 \%$ relative to the fresh material in all cases. Experiments were performed in triplicate and data presented as mean + SEM, $\left({ }^{*} \mathrm{p}=<0.001\right.$ versus control) chorionic and allantoic epithelial layer a part. In the present study we calculated the distance between these two layers (thin stratum) morphometrically in $\mu \mathrm{M}$ after $72 \mathrm{~h}$ of incubation. It is found that thickness of the CAM is considerably increased significantly for $3 \mu \mathrm{g}$ of $\mathrm{HRL}$ $\left({ }^{*} \mathrm{p}=<0.001\right)$ when compared to other two studied concentrations suggestive of its ability of to induce angiogenesis under this concentration. In paraffin-embedded tissues, material shrinkage is estimated to be $\sim 25 \%$ relative to the fresh material and since all tissues were prepared similarly, tissue shrinkage is assumed to be same for all CAM zones. Thus, shrinkage corrections are not necessary while comparing tissue thickness [26].

\section{Angiogenic potential of human recombinant leptin depends on the activation of major angiogenic growth factors - molecular profiling of VEGF 165, VEGF 121, bFGF2, MMP2 and MMP9}

The expression of primary angiogenic growth factors such as VEGF, bFGF2, MMP2 and MMP9 during leptin induced angiogenesis is analysed by measuring the variation in the mRNA level. In our study it is observed that mRNA level expression of these specified angiogenic growth factors increased when treated with HRL (Fig. 5). The intensity of the bands were measured as relative OD and given in Fig. 5b. There was a consistent increase in the expression pattern for $3 \mu \mathrm{g}$ HRL which is significantly higher (*p $=<0.001)$ than that of other two concentrations. It was also observed that for $5 \mu \mathrm{g}$ of HRL the relative mRNA level of VEGF165 and MMP2 increased significantly ( $* \mathrm{p}=<0.001)$ owing to the involvement of these two main angiogenic growth factors on HRL induced angiogenesis. The gene profiling data indicates that the angiogenic ability of HRL mainly depends on the activation VEGF165 and MMP2 since the expression value have increased significantly when treated with 3 and $5 \mu \mathrm{g}$ of HRL. Thus, HRL at $3 \mu \mathrm{g}$ concentration is able to induce maximum angiogenic growth by increasing the gene expression of VEGF121, bFGF2, MMP9 along with VGEF165 and MMP2.

\section{Human recombinant leptin accelerates the gelatinase activity of MMP2}

The potential of HRL to induce neo vascularization was measured intern by its ability to accelerate gelatinase activity of MMP2 to degrade ECM in order to favour ECs proliferation and migration. Figure $6 \mathrm{a}$ indicates that the gelatinase activity of MMP2 is increased indicating ECs proliferation and activation when treated with HRL. The intensity value measured in terms of OD and the graph plotted (Fig. 6b) shows that the gelatinase activity of MMP2 is increased significantly (* $\mathrm{p}=<0.001)$ when treated with 3 and $5 \mu \mathrm{g}$ of HRL. The gelatin zymogram analysis of MMP2 activity demonstrates that HRL has 


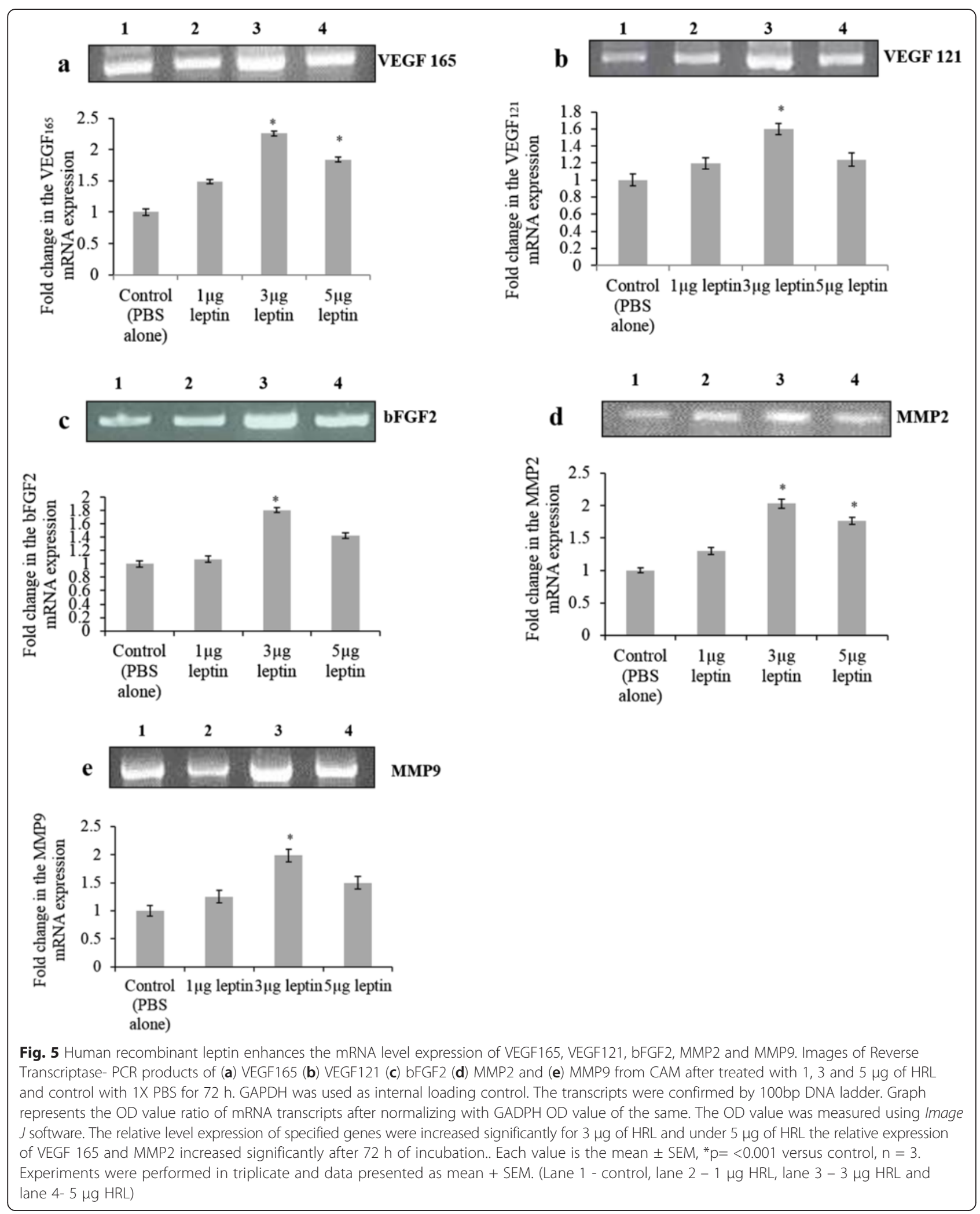

the potential to accelerate neo vascularization by means of increasing the degradation of ECM. This data also indicates that irrespective of the concentration, HRL is able to accelerate the gelatinase activity of MMP2 which in turn signifies that MMP2 plays a major role in HRL induced angiogenesis. 

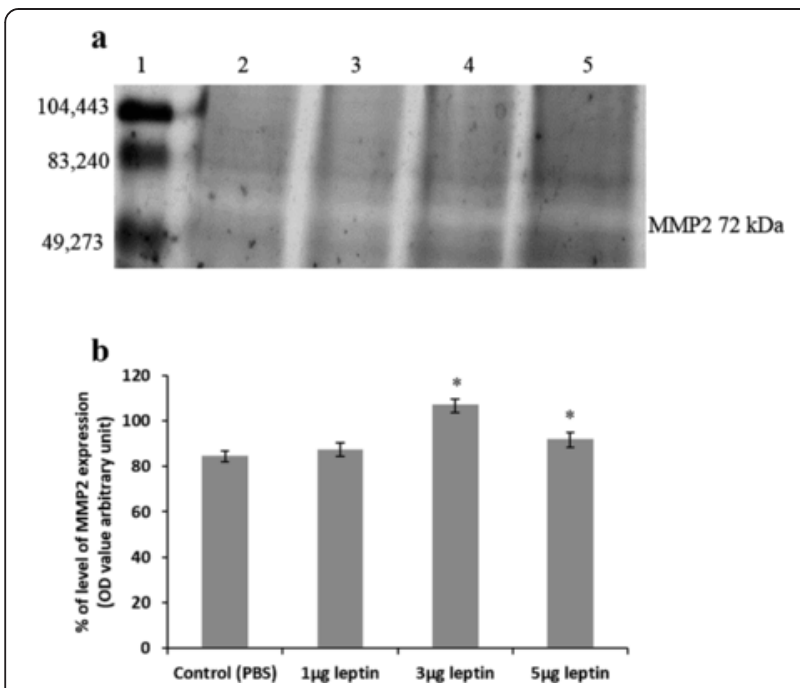

Fig. 6 Human recombinant leptin enhances the gelatinase activity of MMP2. a Image represents the gelatin zymography of MMP2 activity from CAM after treated with 1, 3 and $5 \mu \mathrm{g}$ of HRL and control with $1 X$ PBS for $72 \mathrm{~h}$. The image shows that the gelatinase activity was increased under 3 and $5 \mu \mathrm{g}$ of HRL which is visible as white band against black background. Image is the result of 3 set of experiments. b MMP2 gelatinase activity was measured as OD value using Image I software. Percentage of MMP2 gelatinase activity increased significantly under 3 and $5 \mu \mathrm{g}$ of HRL with higher percentage under $3 \mu \mathrm{g}$ of HRL. Each value is the mean \pm SEM, ${ }^{*} p=<0.001$ versus control, $n=3$. (Lane 1 - protein weight marker, Lane 2 - control, lane 3-1 $\mu \mathrm{g} \mathrm{HRL}$, lane 4-3 $\mu \mathrm{g} \mathrm{HRL}$ and lane 5-5 $\mu \mathrm{g} \mathrm{HRL})$

\section{Human recombinant leptin enhances activation of endothelial cells}

From our data it is clear that the angiogenic potential of HRL is maximum for $3 \mu \mathrm{g}$ concentration enabling the formation of new blood vessels at the treated area. Activation of ECs is considered as one of the major step in angiogenic cascades especially during sprouting angiogenesis [27]. The result obtained from the study confirm the potential of HRL to activate ECs by identifying the presence of CD34 (activated endothelial cell marker) expression on ECs. The result indicates that the CD34 expression is observed at capillary plexus or blood sinus area in which ECs were pooled out along with angioblast cells (Fig. 7b) and the pattern of staining resembles more like coiled structures with elongated protrusions suggestive of the sprouting of ECs under HRL (Fig. 7d). Also the ECs present at the luminal side of the vessels shows higher staining for CD34 than those present in the control (Fig. 7a). Presence of these elongated ECs is suggestive of its enhanced proliferative capacity when treated with HRL which in turn lead to the formation of capillary like structure during the process of angiogenesis especially when sprouting angiogenesis occurs (Fig. 7c and e). In control, those ECs which were present at the lumen of the main vessel shows CD34 expression and no staining was observed at the blood sinus area (Fig. 7a). Thus it can be concluded that HRL has the ability to enhance EC activation which in turn can favour these cells to participate actively in angiogenesis.

\section{Angiogenic potential of human recombinant leptin mainly depends on the activation of VEGF A and MMP2 - protein expression}

Molecular profiling of angiogenic factors after treatment with HRL indicated that angiogenic potential of leptin mainly depends on the functional activation of VEGF A (VEGF 165) and MMP2. Hence we attempted to understand the same by scoring the protein level expression of VEGF A and MMP2 using immunohistochemical method. Results show that with $3 \mu \mathrm{g}$ concentration of HRL the expression of VEGF A protein (Fig. 8b) is higher at the lumen of main vessels as well as at the surrounding small ones as an indicating enhanced ECs activation while control shows the expression only at the luminal surface of the main vessel (Fig. 8a). Expression of MMP2 protein after HRL treatment seems to be more concentrated at the vessel surface particularly at the lumen and also at the thin epithelial layer which contains peptidoglycan extra cellular matrix (Fig. 8d) and this is higher than that of control (Fig. 8c). Presence of increased level of MMP2 protein at the lumen of the vessel is suggestive of the active breakdown of extra cellular matrix in favour of ECs migration and proliferation, support the occurrence of sprouting angiogenesis after HRL treatment. Thus the expression pattern illustrates that HRL able can activate the pool of ECs in such a way that these cells can actively participate in the angiogenic cascade process. The results also demonstrates that $3 \mu \mathrm{g}$ of HRL is capable of inducing neovascularization by increasing the degradation ECM which in turn allows the micro vascular endothelial cells to migrate, proliferate and differentiate. Presence of VEGF A at the endothelium also indicates that $3 \mu \mathrm{g}$ of HRL could favour endothelial cell activation followed by sprouting, initiating new blood vessel formation.

\section{Discussion}

Angiogenesis is defined as the formation of new blood vessels from existing ones which plays an important role in many physiological and pathological events [28]. Leptin, the $\mathrm{Ob}$ gene product is known to exert its biological activity through binding with it receptors termed Ob-R identified in hypothalamus and also in peripheral vascular tissue such as ECs [1, 29]. Reports from various in vivo analyses have suggested both angiogenic and anti angiogenic property of leptin $[3,10]$. But no reports have yielded conclusion results. Reports have also suggested that this dual function of leptin depends on multiple factors such as dose, time of incubation, mode of administration and to certain extent the type of species [3, 4, 8-10, 30]. 


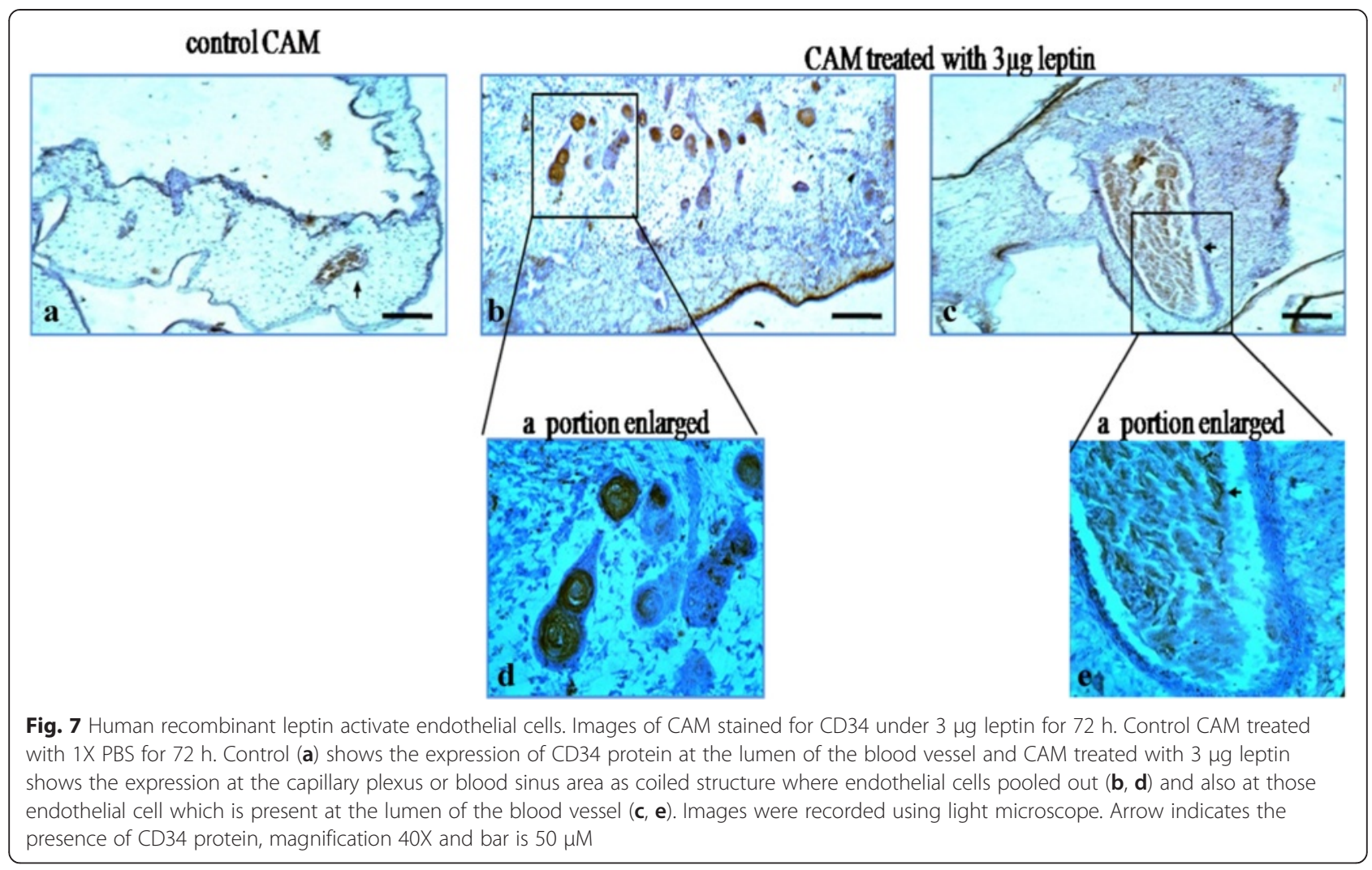

In this context we analysed the angiogenic potential of HRL using in vivo CAM assay. The main advantage of the assay is that it can be used as a rapid method of determining the angiogenic responses because it is considered as an intermediate step between a single model (cell culture) and a more complex system (mammalian model). The other important advantage is that CAM can be used to score the tissue responses towards the angiogenic activity of bio materials accurately and are similar to that of mammalian model responses [31] and also helps in the maintenances of the test materials at the site of administration. To overcome this technical problem we used gelatin sponges for the delivery of the chemical which can held and adhere firmly to the CAM surface with no or less inflammatory reactions [13, 32].

In the present work we found that in ovo administration of HRL is able to induce neovascularization at the treated area in a dose dependent manner. Among those analysed concentration (1,3 and $5 \mu \mathrm{g}$ ) only $3 \mu \mathrm{g}$ of HRL shows significant angiogenic response as observed the increase in vessel growth at an early incubation period of $48 \mathrm{~h}$. The results are in accordance with the earlier research report where the effect was prominent even at $24 \mathrm{~h}$ of incubation [5]. But this shorter time period of incubation on CAM is considered as vasodilation period rather than compound effect [13]. Hence in the present study we performed the experiments for $72 \mathrm{~h}$ to analyse the angiogenic potential of HRL because at this time point newly formed vessels will become stabilized. Interestingly we found that at $72 \mathrm{~h}$ of incubation both 3 and $5 \mu \mathrm{g}$ of HRL shows significant angiogenic effect indicating that the angiogenic potential of HRL not only depends on the concentration but also on the duration of incubation. Microscopic analysis of the growth pattern of the vessels implicates that $3 \mu \mathrm{g}$ of leptin is able to induce more sprouting of new vessels from the existing one when compared to $5 \mu \mathrm{g}$ concentration. Thus, HRL has the potential to induce neovascularization in a dose dependent manner and that $3 \mu \mathrm{g}$ concentration could be more appropriate resulting in earlier and stable angiogenesis.

Placing any foreign material onto CAM can develop inflammatory reaction which can cause secondary vasoproliferative response leading to false positive conclusion [13]. To avoid this issue we have performed a thorough histological evaluation after adding HRL. Changes in the histological structure and altered cellular morphology of the treated area together indicated that HRL is able to initiate new blood vessel formation without local inflammation. HRL of $3 \mu \mathrm{g}$ shows comparatively higher angiogenic responses by accelerating the formation of new vessel through sprouting from the major one. Also the angiogenic or antiangiogenic effect of compound can be effectively analysed by measuring the thickness of the CAM at the treated area as formation and growth of the 


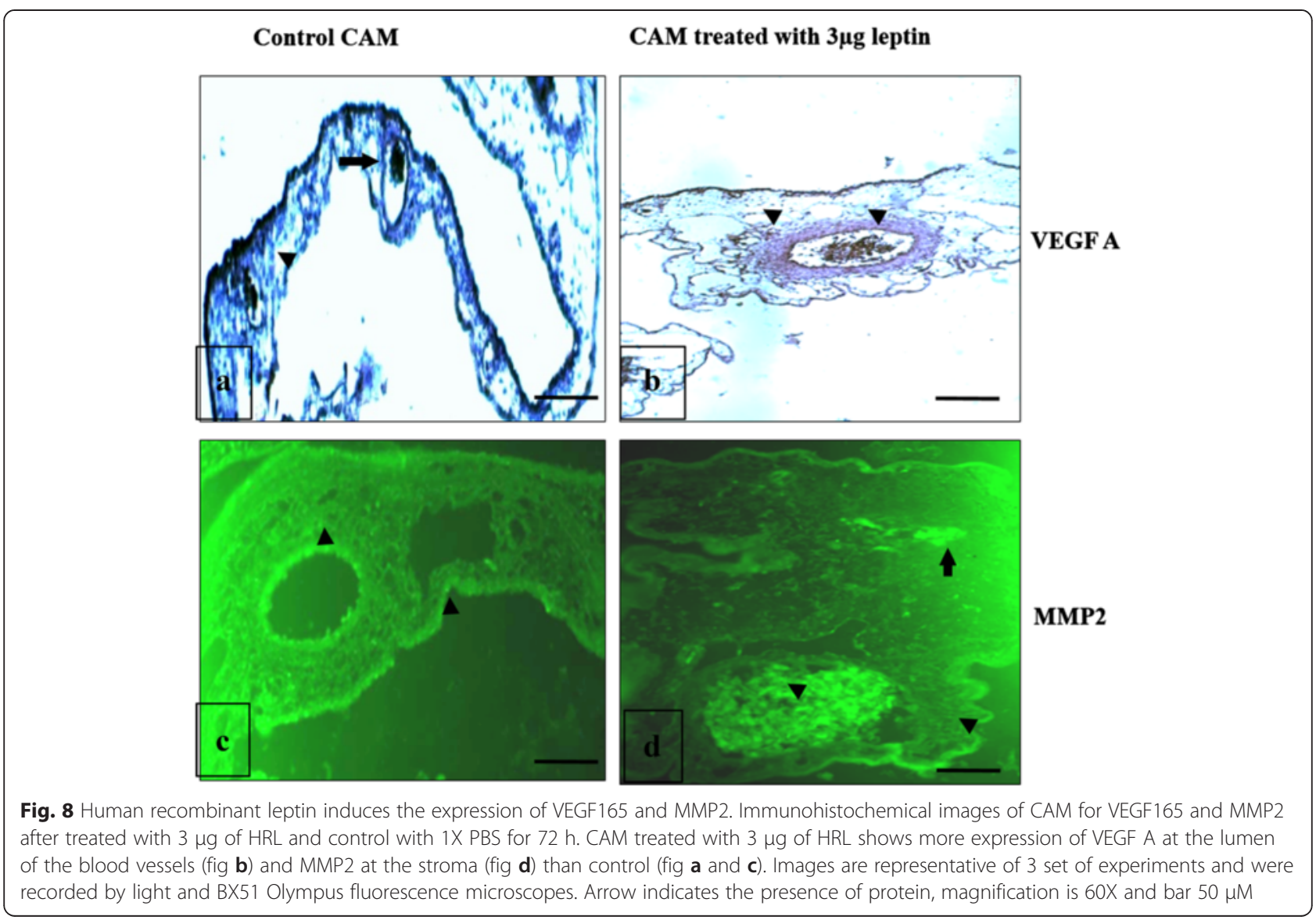

new blood vessels will result in an increased tissue thickness at the stroma region. Any changes related to the growth at the stroma will push chorion and allantoic epithelium a part [15]. In our study we found that $3 \mu \mathrm{g}$ of HRL is able to exert maximum angiogenic response at the stroma region very near to chorionic epithelium rather than mesodermal layer by means of forming capillary like tubes and structures leading to increased tissue thickness.

Results from rat corneal assay as also from various in vitro analyses showed that angiogenic effect of leptin is mediated by VEGF [33] and is supported by matrix remodelling [34]. Molecular profiling of VEGF and its isoforms such as VEGF165 and VEGF121 indicates that HRL induced angiogenesis mainly depends on the expression of VEGF165 rather than VEGF121. Increased mRNA level of MMPs especially MMP2 confirmed the major role of MMP2 in matrix remodelling during HRL induced angiogenesis and is in agreement with the earlier report [29, 34, 35]. Various reports have suggested that the physiological functions of leptin get initiated once it binds to its receptor Ob-R present in various cellular systems. During angiogenesis leptin is shown to exert its activity by binding with $\mathrm{Ob}-\mathrm{R}$ receptor present on ECs followed by up regulation of the downstream signalling [29]. Here also it is possible that the angiogenic response of HRL on CAM is mediated by the receptor $\mathrm{Ob}-\mathrm{R}$ considering the fact that human and chicken leptin receptor has $62 \%$ of homology and the structural domains of the receptor are highly conserved in both [36]. The present data suggests that $3 \mu \mathrm{g}$ of HRL has more angiogenic potential by inducing the increased mRNA level expression of VEGF165, VEGF121, bFGF2, MMP2 and MMP9 and also the protein level expression of VEGF165 and MMP2. Thus of HRL could up regulate the expression of VEGF165 and MMP2 to induce neovascularization resulting in the observed sprouting at the area of treatment.

Activation of EC is considered as one of the most important and preliminary step in the cascade of angiogenesis [28]. In vitro analysis suggested that leptin could initiate the activation of ECs to form tube like structures while inducing angiogenesis [29]. In this work we also analysed the potential effect of HRL on the activation of ECs by studying the immuno localization of CD34 expression on CAM ECs. Presence of more activated ECs at the blood sinus region along with coiled like structures formation of ECs at the sinus area together highly support the ability of HRL to activate ECs during angiogenesis. Presences of elongated ECs at the lumen of the 
vessel also indicates that $3 \mu \mathrm{g}$ of HRL could be the optimum concentration that can initiate the formation of new capillary like structures which is remarkable at the first phase of sprouting angiogenic process.

\section{Conclusion}

The present work demonstrates that HRL has the potential to induce neovascularization by means of sprouting at the treated area. Expression of CD34 on activated ECs indicates that HRL can initiate the activation ECs and could favour the formation of tube like structure from the pool of ECs. Increased tissue thickness and altered cellular morphology of CAM with HRL treatment supports dose dependent angiogenic ability of HRL. It was also observed that HRL induced angiogenesis mainly depends on the activation of VEGF165 and MMP2. Direct visualization and growth of newly formed vessels at the treated area demonstrates that the angiogenic ability especially sprouting effect of HRL depends on the concentration and time of incubation. It is found that $3 \mu \mathrm{g}$ of HRL exhibits significant angiogenic response at $72 \mathrm{~h}$ of incubation. Altogether our findings suggest that HRL could be a useful angiogenic therapeutic molecule and can be more effectively used in the future especially for the treatment of ischemic disorders and wound healing which requires new vessel formation.

\section{Competing interests}

The authors declare that they have no competing interests.

\section{Authors' contributions}

R M carried out the experimental studies and made manuscript. M R coordinated and helped in interpreting the data and reviewing the manuscript. Both authors read and approved the final manuscript.

\section{Acknowledgement}

We would like to thank Dr. Shanthi, MBBS, MD (Pathology), Department of Pathology for immunohistochemical study and Mr. Thankaraj for microscope technical assistant. We would like to thank Dr. Li Haiqing, MD, Ph.D, -Technology transfer specialist, National Cancer Institute, Rockville, USA for the kind gift of Rabbit polyclonal MMP2 antibody. We thank the funding agency, University Grant commission of India for giving merit fellowship and also for supporting the department under SAP program.

Received: 18 February 2015 Accepted: 2 June 2015

Published online: 10 June 2015

\section{References}

1. Zhang Y, Proenca R, Maffei M, Barone M, Leopold L, Friedman JM. Positional cloning of the mouse obese gene and its human homologue. Nature. 1994;372:425-32.

2. Goetze S, Bungenstock A, Czupalla C, Eilers F, Stawowy P, Kintscher U, et al. Leptin induces endothelial cell migration through akt, which is inhibited by ppargamma-ligands. Hypertension. 2002;40:748-54.

3. Stavros A, Anastasios J, Karayiannakis, Maria L, Anna E, Alexandros P, et al. Human leptin induces angiogenesis in vivo. Cytokine. 2008;42:353-7.

4. Hyun-YP, Hyuck MK, Hyun JL, Bum KH, Ju YL, Byoung EP, et al. Potential role of leptin in angiogenesis: leptin induces endothelial cell proliferation and expression of matrix metalloproteinases in vivo and in vitro. Exp Mol Med. 2002;33:95-102.

5. Bouloumie A, Drexler HC, Lafontan M, Busse R. Leptin, the product of $\mathrm{Ob}$ gene, promotes angiogenesis. Circ Res. 1998;83:1059-66.
6. Sierra- Honigmann MR, Nath AK, Murakami C, Garcia- Cardena G, Papapetropoulos A, Sessa WC, et al. Biological action of leptin as an angiogenic factor. Science. 1998;281:1683-6.

7. Islami D, Bischof $P$, Chardonnes D. Modulation of placental vascular growth endothelial growth factor by leptin and hCG. Mol Hum Reprod. 2003;9:395-8.

8. Vyboh P, Zeman M, Bilcik B, Sarnikova B, Kostal L. Angiogenic effect of leptin in the quail chorioallantoic membrane. Acta Vet Brno. 2010;79:13-7.

9. Ribatti D, Nico B, Belloni AS, Vacca A, Ronacali L, Nussdoefer GG. Angiogenic activity of leptin in the chick embryo chorioallantoic membrane is in part mediated by endogenous fibroblast growth factor-2. Int J Mol Med. 2001;8:265-8.

10. Sua L, Raoa K, Guoa F, Lia X, Ahmeda AA, Nia Y, et al. In ovo leptin administration inhibits chorioallantoic membrane angiogenesis in female chicken embryos through the STAT3- mediated vascular endothelial growth factor (VEGF) pathway. Domest Anim Endocrinol. 2012;43:26-36.

11. Stefan F, Birgit S, Heiko K, Nicole K, Josef P. Leptin enhances wound re-epithelialization and constitutes a direct function of leptin in skin repair. J Clin Invest. 2000;106:501-9.

12. Nowak SP, van Beijnum JR, van Berkel $M$, van den Bergh $H$, Griffioen AW Vascular regrowth following photodynamic therapy in the chicken embryo chorioallantoic membrane. Angiogenesis. 2010;13:281-92.

13. Ribatti D, Nico B, Vacca A, Roncali L, Burri PH, Djonov V. Chorioallantoic membrane capillary bed: A useful target for studying angiogenesis and anti-angiogenesis in vivo. Anat Rec. 2001;264:317-24.

14. Reji BR, Karthick R, Malathi R. Angiogenic efficacy of Heparin on chick chorioallantoic membrane. Vascular Cell. 2012;4:1-8.

15. Niemisto A, Dunmire V, Yli-Harja O, Zhang W, Shmulevich I. Robust quantification of in vitro angiogenesis through image analysis. IEEE Trans Med Imaging. 2005;24:549-53.

16. Verma K, Gu J, Werner E. Tumor Endothelial Marker 8 Amplifies Canonical Wnt Signaling in Blood Vessels. PLoS One. 2011;6:223-34.

17. Ribatti D. Chick embryo chorioallantoic membrane as a useful tool to study angiogenesis. Int Rev Cell Mol Biol. 2008;270:181-224.

18. Ribatti $\mathrm{D}$, Presta $\mathrm{M}$. The role of fibroblast growth factor-2 in the vascularization of the chick embryo chorioallantoic membrane. J Cell Mol Med. 2002;6:439-46.

19. Yang EY, Moses HE. Transforming Growth factor Beta -1 induced changes in cell migration, Proliferation and Angiogenesis in the Chicken chorioallantoic Membrane. J Cell Biol. 1990;111:731-41.

20. Giannopoulou E, Papadimitriou E. Amifostine has antiangiogenic properties in vitro by changing the redox status of human endothelial cells. Free Radic Res. 2003;37:1191-9.

21. Larger E, Marre M, Corvol P, Gasc JM. Hyperglycemia-induced defects in angiogenesis in the chicken chorioallantoic membrane model. Diabetes. 2004;53:752-61.

22. Kim DH, Lilliehook C, Roides B, Chen Z, Chang M, Mobashery S, et al. Testosterone-induced matrix metalloproteinase activation is a checkpoint for neuronal addition to the adult song bird brain. J Neurosci. 2008;28:208-16.

23. Zijlstra A, Aimes RT, Zhu D, Regazzoni K, Kupriyanova T, Seandel M, et al. Collagenolysis-dependent angiogenesis mediated by matrix metalloproteinase-13 (collagenase-3). J Biol Chem. 2004;279:27633-45.

24. Liekens S, De Clercq E, Neyts J. Angiogenesis: regulators and clinical applications. Biochem Pharmacol. 2001;61:253-70.

25. Ozyigit MO, Kahraman MM, Sonmez G. The identification of matrix metalloproteinase and their tissue inhibitors in broiler chickens by immunohistochemistry. Avian Pathol. 2005;34:509-16.

26. Arava $\mathrm{R}$, llan $\mathrm{H}$, Amos A. Regional and developmental variations of blood vessel morphometry in the chick embryo chorioallantoic membrane. J Exp Biol. 2005;208:2483-8.

27. Ha Y, Tsukada A, Saito N, Shimada K. Changes in mRNA expression of $\mathrm{mmp}-2$ in the mullerian duct of chicken embryo. Gen Comp Endocrinol. 2004;139:131-6.

28. Klagsbrun M, D'Amore PA. Regulators of angiogenesis. Annu Rev Physiol. 1991;53:217-39.

29. Park HY, Kwon HM, Lim HJ, Hong BK, Lee JY, Park BE, et al. Potential role of leptin in angiogenesis, Leptin induces endothelial cell proliferation and expression of matrix metalloproteinases in vivo and in vitro. Exp Mol Med. 2001;33:95-102

30. Aronis KN, Diakopoulos KN, Firenzia CG, Chanberland JL, Mantzoros CS Leptin administered in physiological or pharmacological doses does not regulate circulatory angiogenic factors in human. Diabetol. 2011;54:2358-67. 
31. Valdes TI, Kreutzer D, Moussy F. The chick chorioallantoic membrane as a novel in vivo model for the testing of biomaterials. J Biomed Mater Res. 2002;62:273-82.

32. Ribatti D, Gualandris A, Bastaki M, Vacca A, lurlaro M, Roncali L, et al. New model for the study of angiogenesis and antiangiogenesis in the chick embryo chorioallantoic membrane: The gelatin sponge/chorioallantoic membrane assay. J Vasc Res. 1997;34:455-63.

33. Rahmouni K, Haynes WG. Endothelial effects of leptin: Implications in health and diseases. Curr Diab Rep. 2005;5:260-6.

34. Cao R, Brakenhielm E, Wahlestedt C, Thyberg J, Cao Y. Leptin induces vascular permeability and synergistically stimulates angiogenesis with fgf-2 and vegf. Proc Natl Acad Sci U S A. 2001;98:6390-5.

35. Newby AC. Dual role of matrix metalloproteinases (matrixins) in intimal thickening and atherosclerotic plaque rupture. Physiol Rev. 2005;85:1-31.

36. Horev G, Einat P, Aharoni T, Eshdat Y, Friedman EM. Molecular cloning and properties of the chicken leptin-receptor (CLEPR) gene. Mol Cell Endocrinol. 2000;162:95-106.

\section{Submit your next manuscript to BioMed Central and take full advantage of:}

- Convenient online submission

- Thorough peer review

- No space constraints or color figure charges

- Immediate publication on acceptance

- Inclusion in PubMed, CAS, Scopus and Google Scholar

- Research which is freely available for redistribution 